FERMILAB-TM-1951

\title{
Neutrino Beams Using the Main Injector
}

\author{
A.J. Malensek \\ Fermi National Accelerator Laboratory \\ P.O. Box 500, Batavia, Illinois 60510
}

November 1995 


\section{Disclaimer}

This report was prepared as an account of work sponsored by an agency of the United States Government. Neither the United States Government nor any agency thereof, nor any of their employees, makes any warranty, expressed or implied, or assumes any legal liability or responsibility for the accuracy, completeness, or usefulness of any information, apparatus, product, or process disclosed, or represents that its use would not infringe privately owned rights. Reference herein to any specific commercial product, process, or service by trade name, trademark, manufacturer, or otherwise, does not necessarily constitute or imply its endorsement, recommendation, or favoring by the United States Government or any agency thereof. The views and opinions of authors expressed herein do not necessarily state or reflect those of the United States Government or any agency thereof. 


\title{
Neutrino Beams Using the Main Injector
}

\author{
Anthony J. Malensek
}

October 12, 1995

\section{Introduction}

TM-1946[1] summarizes the status of the NuMI project. This note presents more details on the technical design of the various NuMI beams. Several beamline options are investigated for producing neutrinos--( 1 ) a wide-band beam(WBB) using a Double Horn, (2) a beam using only a single Lithium Lens, and (3) a two stage narrow-band beam(NBB) using a Lithium Lens, quadrupoles and dipoles. The first two are designed to maximize the muon neutrino event rate; the third is designed to have a tunable range of parent momenta from 5-45 GeV/c. In the context of NuMI, the Double Horns and its target were concepts first described[2] in 1991. The Lithium Lens has been used at Fermilab for pbar production for several years. With recent upgrades[3], it forms the basis of what will be used by NuMI. Narrow band beams using conventional dipoles and quadrupoles have been studied, but have less acceptance than one using a lithium lens.

The following practical limits are imposed on each of the systems:

(1) Horns: the necks will not have a smaller radius than $1 \mathrm{~cm}$; the maximum current will not exceed $170 \mathrm{kAmp}$. Keeping the inside diameter large allows the primary proton beam to vary in position, yet not strike the fragile neck. In addition, there is a trade-off between decreasing the radius and increasing the wall thickness to maintain the required strength of the conductor material.

(2) Magnets: reasonable conventional designs are used. The maximum gradient for quadrupoles is $12 \mathrm{kG} /$ half-aperture; the maximum field for dipoles with a 2 inch full gap is about $16 \mathrm{kG}$; larger apertures scale the field down. Although not a primary consideration, it is desirable for the magnification in each plane to be comparable (within a factor of 2 or 3 is $\mathrm{OK})$.

(3) Lithium Lens: the maximum radius is $1.0 \mathrm{~cm}$ with a maximum gradient of $100 \mathrm{kG} / \mathrm{cm}$.

(4) Dumps: at the place where the primary protons are absorbed, the transverse beam center is $\approx 1$ inch off the edge of the acceptance; the minimum length for a primary beam dump is 12 feet. 


\section{Horns}

The nominal design has a system with Horn\#1 being 4 meters long and Horn\#2 being 3 meters long. For purposes of construction and handling, it is desirable to have horns shorter in length. This may also have an advantage with respect to the total amount of shielding needed around the elements in the Target Hall. It is almost a certainty that the 4 meter horn cannot be manufactured in one piece. Furthermore, from a structural point of view, it is desirable to have the neck at one end of the horn, not in the middle. I have therefore looked at the possibilities for making a WBB with shorter horns and they are listed in the Table below. For purposes of identification, the horn systems carry a name having the total length of both horns (i.e., H5 is a 2 meter Horn\#1 followed by a 3 meter Horn\#2).

\section{Table 1}


All horn designs use the long segmented carbon target $(\mathrm{r}=2 \mathrm{~mm}, 100$ $\mathrm{cm}$ graphite, $56 \mathrm{~cm}$ total gap) discussed in TM-1946 and all have the neck at the downstream end, except H7. Using a coordinate system which has $\mathrm{Z}=0$. as the upstream end of Horn\# 1 , the target begins at $\mathrm{Z}=-1.6$ meters. Both horns use the same current as the nominal design, $170 \mathrm{kAmps}$ each. At this time the length of the muon shield from the dump to the experimental hall has not been fixed, pending the outcome of GEANT runs. For purposes of running the program NUADA[4] for a comparison between the various length horns, I have used a length of 250 meters, which should be conservative. The other parameters of interest are, (a) decay length $=800$ meters, $($ b) decay pipe radius $=1$ meter, $(\mathrm{c})$ distance to the long baseline detector $=732 \mathrm{~km}$, (d) radius of the long baseline detector $=10$ meters, (e) radius of the short baseline detector $=0.9$ meters. One year of running is taken to be 3.7E20 incident protons.

Although the list is not exhaustive, it indicates the general trade off with flux and events as the length of the horn system is reduced. A comparison of the NuMu event rate for horn systems of different lengths is shown in Figure 1 for the short baseline and in Figure 2 for the long baseline. Absorption in the horn conductors has been taken into account.

One can notice that the "shoulder" around $20 \mathrm{GeV}$ is most pronounced for H7, compared to all the others. For the most part, this comes from the "vee" shape of H7; the others could be described as "half vee".

An interesting property of the various horn systems is their depth of field. This can be thought of as a correlation between the spectrum in the detector and the Z position of the target. Since the conical shapes of the horns are designed to focus a certain pt, secondary particles coming from the "front" of the target will produce higher energy neutrinos than those from the "back" of the target. The depth of field not only determines the energy, but also the relative number; the weighting will be greatest from the upstream end of the target, because that's where the number of protons is greatest. The depth of field for each horn system is shown in Figure 3 and Figure 4 for the short and long baselines. The depth of field for a horn system(Horn\#1 and Horn\#2) is obtained by comparing the event rate at the detector while moving a one-quarter interaction length target in Z. All curves are normalized with respect to the nominal horn system (i.e., 1.0 is the highest point of $\mathrm{H} 7$ at $\mathrm{Z} \approx-0.5$ meter).

For ease of construction, handling, and production, a one interaction length target placed at the peak of the depth of field would be adequate. However in the case of NuMI, all the non-interacting protons travel down the decay pipe to the dump where they create background from charm decay. For the short baseline experiment it is therefore desirable to have a long target to interact as many protons as possible. The long target also gives a slightly higher event rate. NUADA results for a single one interaction length target 
and the nominal( $2 \lambda$ plus gaps) show about a $30 \%$ decrease in $\mathrm{NuMu}$ events.

\section{$3 \quad$ Single Lithium Lens}

A lithium lens is a cylinder of solid lithium carrying current so that a particle passing through the lithium at an angle with respect to the lens axis will experience a radial Lorentz force. A lens with radius $\mathrm{r}_{\mathrm{O}}$, length $\mathrm{L}$ carrying a current $\mathrm{I}$, will produce an azimuthal magnetic induction $\mathrm{B}(\mathrm{r})=\mu_{\mathrm{O}} \mathrm{Ir} / 2 \pi \mathrm{r}_{\mathrm{O}}{ }^{2}$. An ideal lens will make a beam parallel when the distance from a point source to the upstream face of the lens is $\mathrm{d}=1 /(\mathrm{k}$ $\tan (\mathrm{kL}))$ where $\mathrm{k}=(0.03 \mathrm{G} / \mathrm{p})^{1 / 2}, \mathrm{~L}=$ length of lens(meter), $\mathrm{G}=$ lens $\operatorname{gradient}(\mathrm{kG} / \mathrm{m})$, and $\mathrm{p}=$ particle momentum $(\mathrm{GeV} / \mathrm{c})$.

The lens used for pbar collection at Fermilab has a radius of $1 \mathrm{~cm}$ and a length of $15 \mathrm{~cm}$. Outside the $2 \mathrm{~cm}$ diameter is a thin water cooled container which is encased inside a steel cylinder having cooling tubes. The point is that particles traveling through the lithium are absorbed slightly, while particles at radii larger than $1 \mathrm{~cm}$ get completely absorbed. The upgraded lens[3] is designed to produce a peak magnetic gradient of $100 \mathrm{kG} / \mathrm{cm}$ with high reliability. The single lithium lens was optimized for event rate by fixing the gradient at this highest value and adjusting the distance between the target and the lens. Since the lens is short, it is important to keep the length of the target short to minimize the divergence of various momenta. NUADA results analogous to the horn runs for the optimized single lithium lens is shown in Figure 5 and Figure 6 for the short and long baselines. The center-of-target to beginning of lens distance is optimum at about $75 \mathrm{~cm}$. This corresponds to ideal point to parallel focusing for about $35 \mathrm{GeV} / \mathrm{c}$. Absorption in the lithium has been taken into account and is about an $8 \%$ effect.

\section{$4 \quad$ Lithium Lens with Quadrupoles and Dipoles}

The NBB beam follows a different trajectory than the horn beam. To make the final beam position and direction the same after it passes through either the horn or a bending dichromatic, compensating bends are needed in the primary proton beam. A possible solution that puts the NBB on the same final trajectory as the WBB was given in TM-1946.

The proposed NBB design uses a lithium lens in the first stage and dipoles, quadrupoles and collimators in the second stage. By itself, the lithium lens produces a large angular divergence as well as a large momentum acceptance. The second stage is added to select $\mathrm{dp} / \mathrm{p}$, to dump 
the primary protons, and to decrease the angular divergence. The schematic of the design is given in Figure 7. The optics consist of:

(1) point to parallel--target through lithium lens,

(2) parallel to point--first doublet to momentum slit,

(3) point to parallel--momentum slit through 2 nd doublet.

The first bending magnet separates the secondary beam from the primary protons and also gives a dp/dx correlation at the momentum slit. Even at $60 \mathrm{GeV} / \mathrm{c}$, the primary protons are separated from the secondaries by more than an inch outside the acceptance. The second bending magnet, along with the quadrupole near the slit recombine the momenta. The dipoles bend in the same direction giving a total of $14 \mathrm{mr}$. To keep the wide-band background small, it is important to have an insert in the dipole following the target that collimates the beam. It also serves to limit the radiation on the downstream magnets.

The NBB can be tuned to various momenta, by changing the focal length of the lithium lens, keeping its gradient constant, and then scaling the currents in the dipoles and quadrupoles. This retains the highest acceptance while moving only the target position, keeping the lithium lens and downstream magnets at fixed positions. The optics of the primary protons are adjusted to move the waist in $\mathrm{Z}$ as the target location moves. Tunes for 30,45 and $60 \mathrm{GeV} / \mathrm{c}$ parent particles have a center-of-target to upstream end of the lithium lens distance of $1.985 \mathrm{ft} .3 .063 \mathrm{ft}$. and $4.141 \mathrm{ft}$ respectively. Since the depth of field for a lithium lens is very short compared to the Double Horn System, the NBB target drill be different from the WBB design. It will be a shorter and higher density--about one interaction of nickel, like the Fermilab pbar target.

NUADA runs at 30, 45 and $60 \mathrm{GeV} / \mathrm{c}$ give the muon neutrino event rates and are plotted in Figure 8 for the long baseline. Absorption in the lithium has been taken into account. At this time only the long baseline has expressed an interest in NBB running, however for completeness, the $\mathrm{NuMu}$ total event rates for the short baseline are 4.07E5, 4.50E5 and $2.87 \mathrm{E} 5$ at 30,45 and $60 \mathrm{GeV} / \mathrm{c}$ tunes.

\section{Appendix A}

NUADA has been modified to put in more accurate values for the total(includes quasi-elastics) muon neutrino cross section when it converts flux to events. The default value of $0.67 * \mathrm{E}$ is low by more than $10 \%$ for energies below $10 \mathrm{GeV}$, and is over $30 \%$ low at $1 \mathrm{GeV}$. Table 2 lists the cross sections [5] versus energy that has been used to calculate the event rates. Graphically the NuTau/NuMu ratio is shown in Figure 9. The $\mathrm{NuTau} / \mathrm{NuMu}$ ratio of cross sections for maximal mixing $\left[\sin ^{2}(2 \theta)=1\right.$.] is $1 / 2$ for the average oscillation probability. The values plotted are therefore 
$1 / 2$ those listed in reference[6]. Adding the quasi-elastics will make a slight increase in the ratio.

It is important to run NUADA with more than one bin into which the target is divided(in Z). Otherwise there may be steep fall-offs in the flux as a function of energy. I have typically run with five divisions for the NBB, and 12 divisions for the WBB; i.e., the line in the file has "TARGET 5".

\section{References}

[1] D. A. Crane et al., "Status Report: Technical Design of Neutrino Beams for the Main Injector (NuMI)." (Batavia, IL.: Fermilab Internal Report TM-1946, 1995).

[2] R. Bernstein et al., "Conceptual Design Report: Main Injector Neutrino Program." (Batavia, IL.: Fermilab Internal Report version 1.1, June 14, 1991).

[3] S. O'Day and K. Anderson, "Electromagnetic, Thermal and Structural Analysis of the Fermilab Antiproton Source Lithium Lens." (Dallas, TX.: Proceedings of the 1995 IEEE PAC Conference).

[4] D. C. Carey and V. A. White, "NUADA, The Fermilab Neutrino Flux Program." (Batavia, IL.: Fermilab Internal Report, June 1,1975). See modifications in Appendix A.

[5] The NuMu total cross section values are from Soudan 2. H. Gallagher, (Batavia, IL.: Fermilab Internal Report NuMI-112, 1995). Basically this uses two data sets, one from low energy and the other from high energy; $1-10 \mathrm{GeV}$ is from N. J. Baker et al., Physics Rev. D 25, 617(1982), and from 40-250 GeV from R. Blair et al., Phys. Rev. Letters $51,343(1983)$.

[6] B. P. Roe, "Calculation of Tau Neutrino Cross Sections." (Ann Arbor, Michigan: University of Michigan Internal Report UM-HE94-10, June $1,1994)$. 
Short Baseline Event Rate

for Various Horn Systems

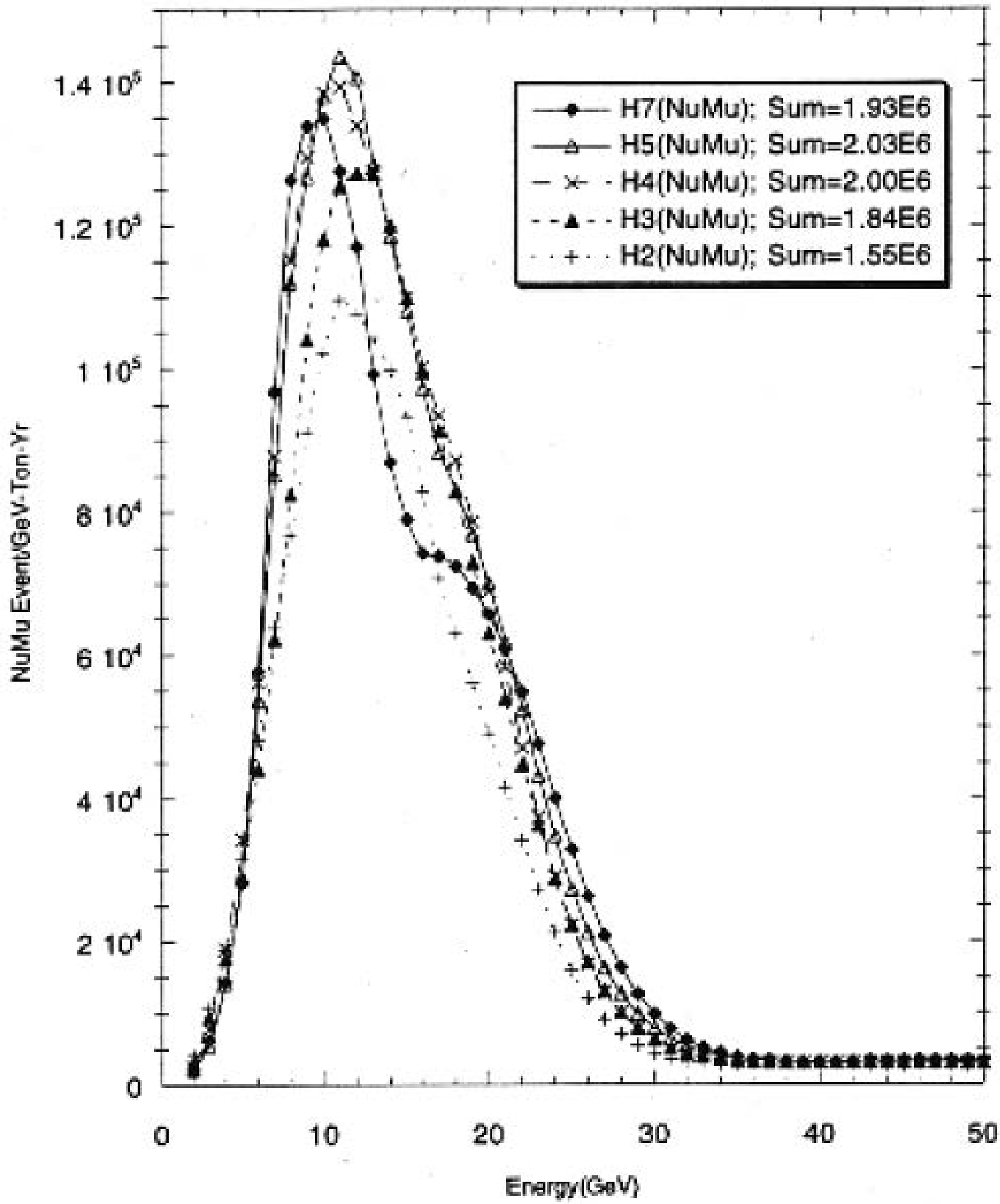

Figure 1 


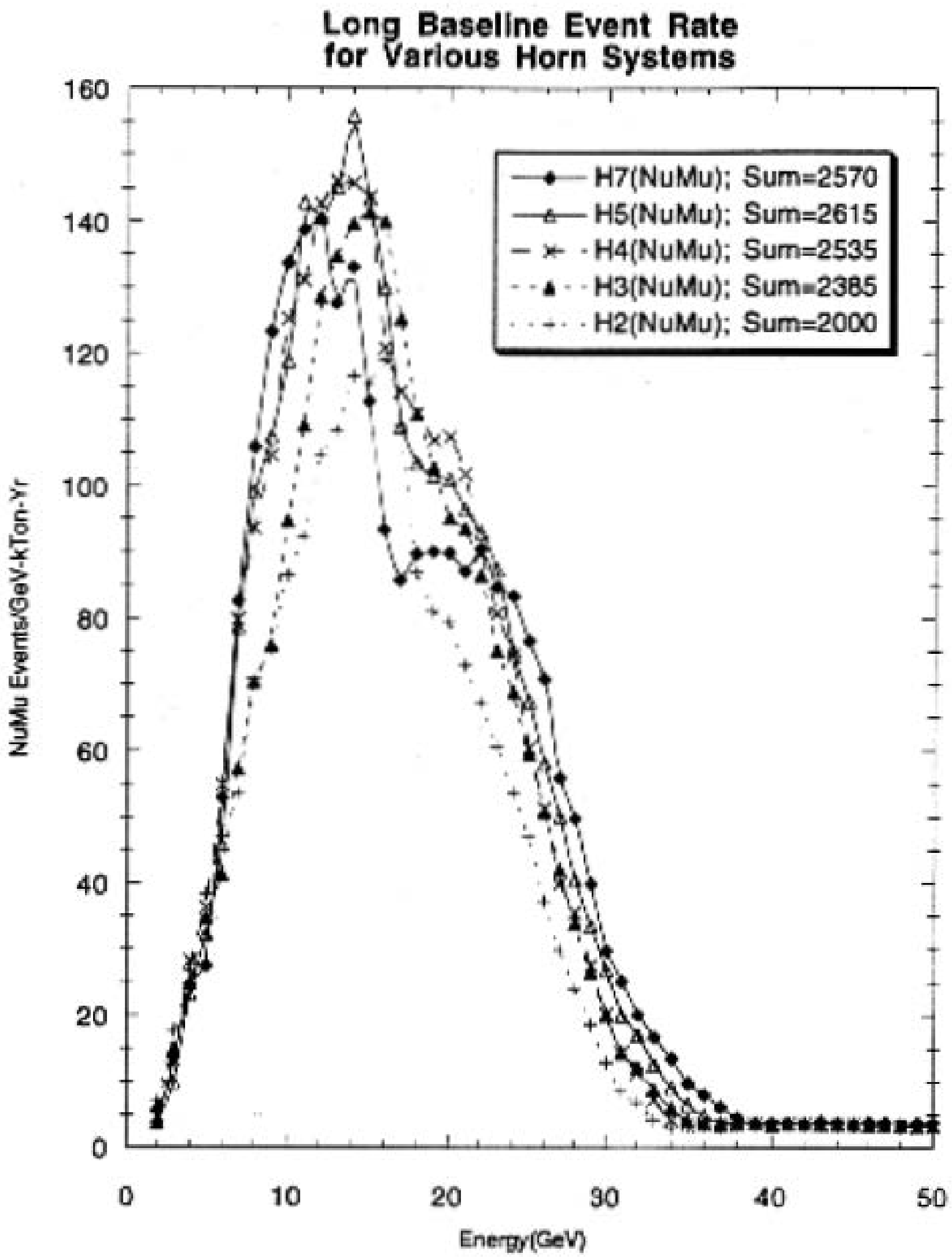

Figure 2 
Depth of Field for Various Horn Systems; Short Baseline

( $Z=0$ is upstream end of Horn\#1)

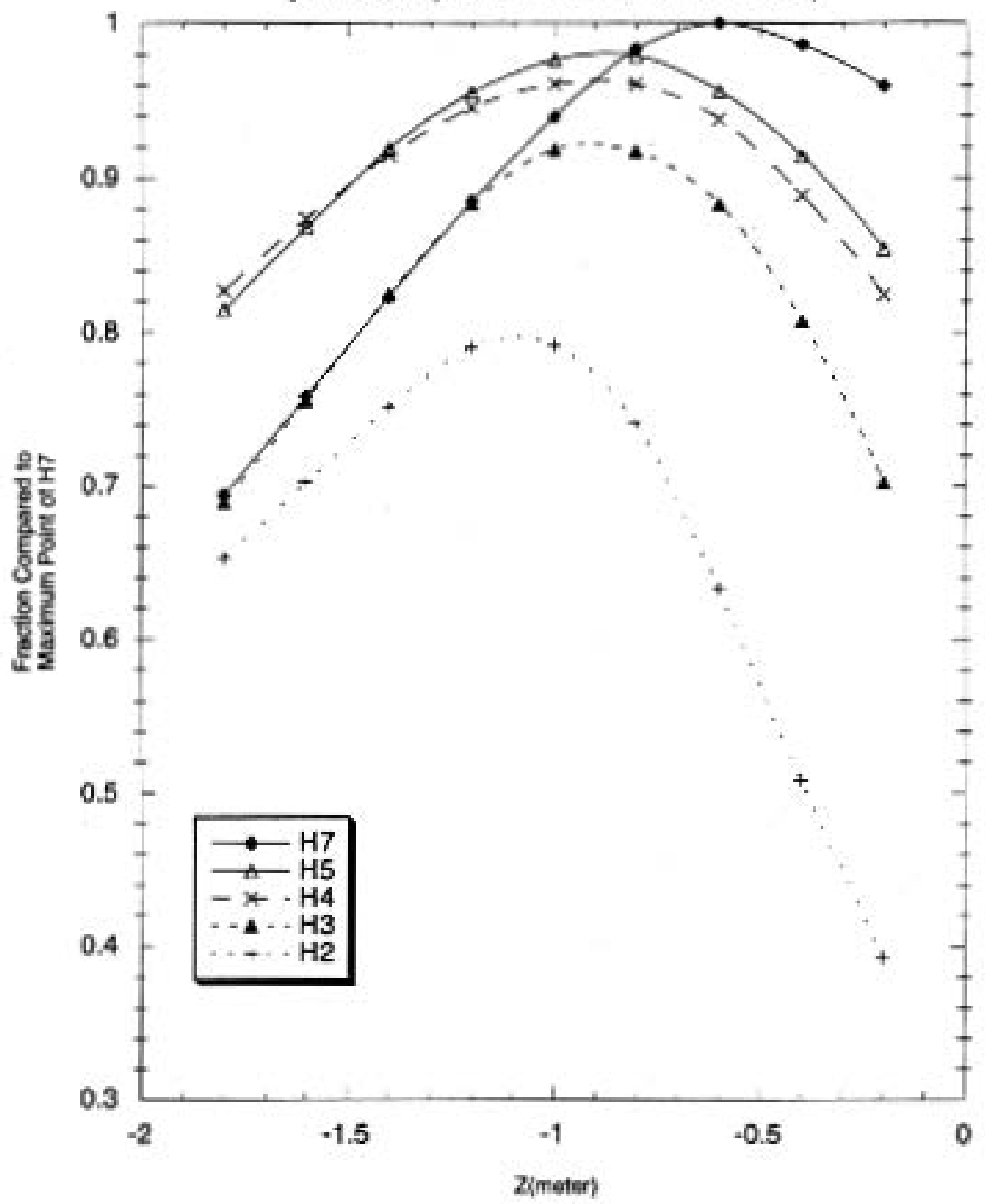

Figure 3 
Depth of Field for Varlous Horn Systems; Long Baseline

( $Z=0$ is upstream end of Horn\#1)

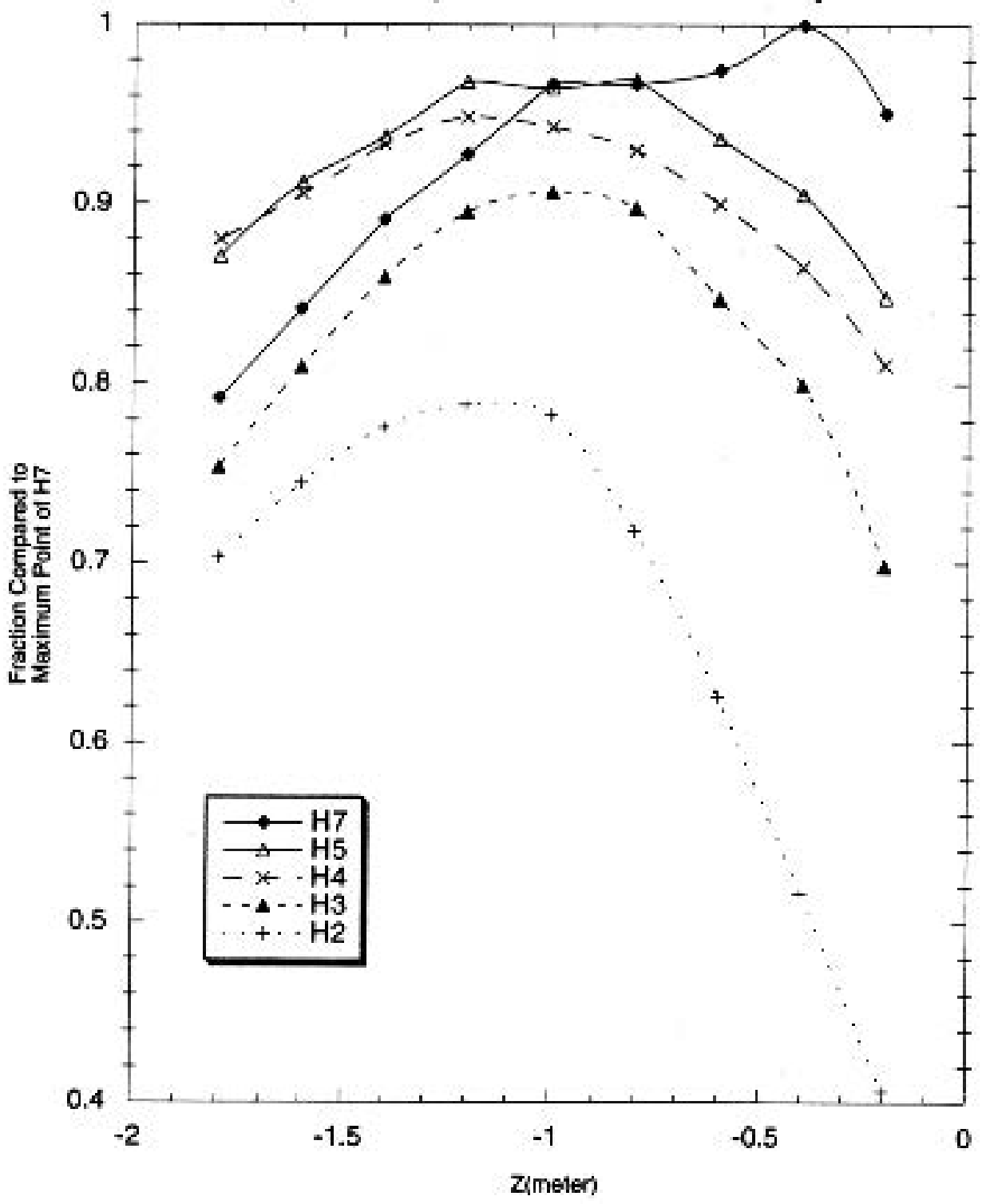

Figure 4 
Short Baseline Event Rate

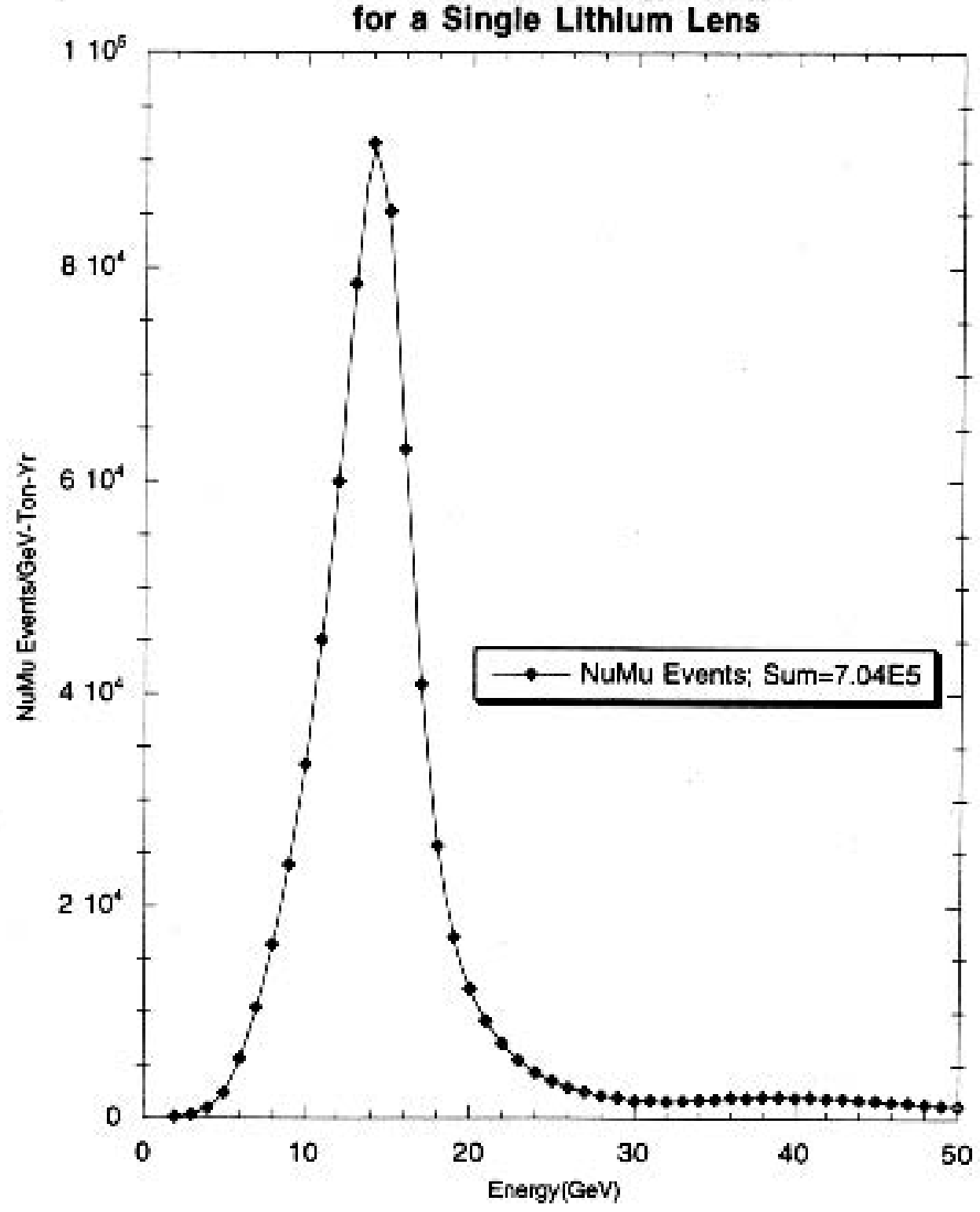

Figure 5 


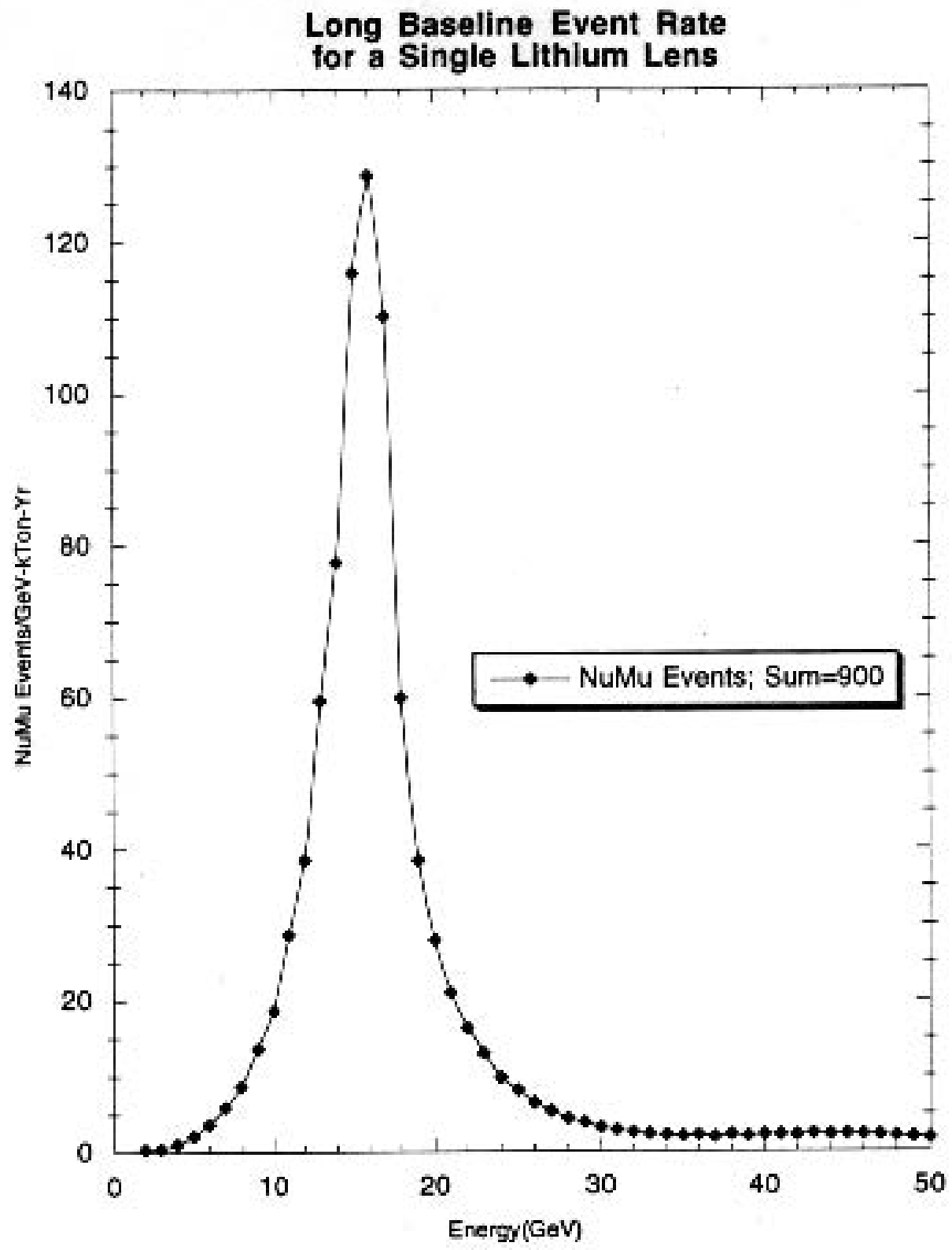

Figure 6 


\section{NBB OPTICS}

\section{$\mathrm{r}=1 \mathrm{~cm}$ Lens}

(Not to Scale)

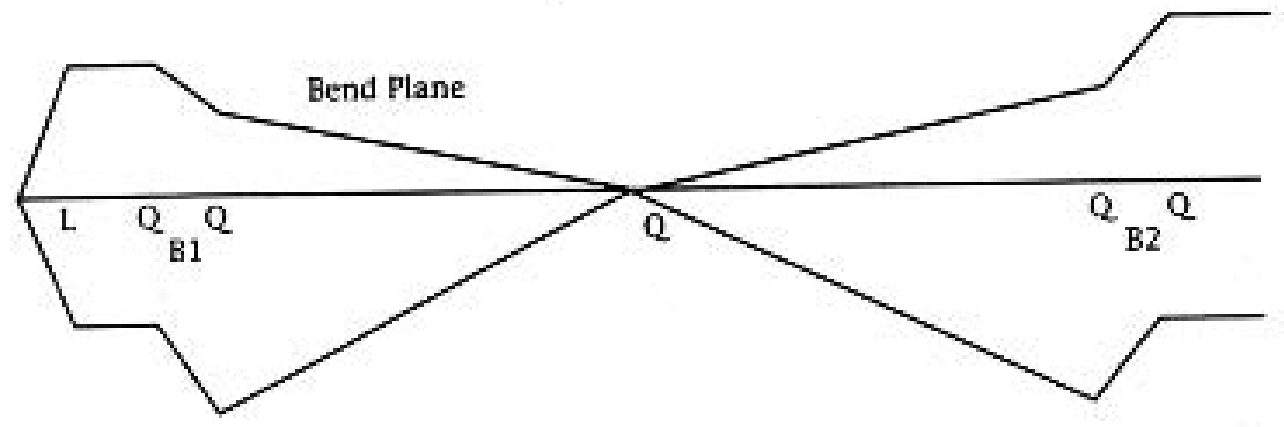

Figure 7

$\mathrm{NBB}$ at $45 \mathrm{GeV}$

Center of Target

Drift

Lithium Lens

Drift

Quad

Drift

Bend

Drift

Quad

Drift

Variable Slit(Dump)

Quad

Drift

Quad

Drift

Bend

Drift

Quad
$3.063 \mathrm{ft}$

$0.500 \mathrm{ft}$

$12.0 \mathrm{ft}$

$4.00 \mathrm{ft}$

$3.00 \mathrm{ft}$

$3.25 \mathrm{ft}$

$3.00 \mathrm{ft}$

$4.00 \mathrm{ft}$

$39.0 \mathrm{ft}$

Aperture- $3.5 \mathrm{~cm}$

$4.00 \mathrm{ft}$

52. $0 \mathrm{ft}$

$4.00 \mathrm{ft}$

$3.00 \mathrm{ft}$

$3.25 \mathrm{ft}$

$3.00 \mathrm{ft}$

$4.00 \mathrm{ft}$
$100 \mathrm{kG} / \mathrm{cm}$

$+1.54322 \mathrm{kG} / \mathrm{cm}$

$+11.2407 \mathrm{kG}$

$-2.00832 \mathrm{kG} / \mathrm{cm}$

$+1.54344 \mathrm{kG} / \mathrm{cm}$

$-1.79131 \mathrm{kG} / \mathrm{cm}$

$+10.1100 \mathrm{kG}$

$+1.43495 \mathrm{kG} / \mathrm{cm}$

Quadrupole apertures have $\mathrm{r}=5 \mathrm{~cm}$; bends are $10 \mathrm{~cm} \times 10 \mathrm{~cm}$. 


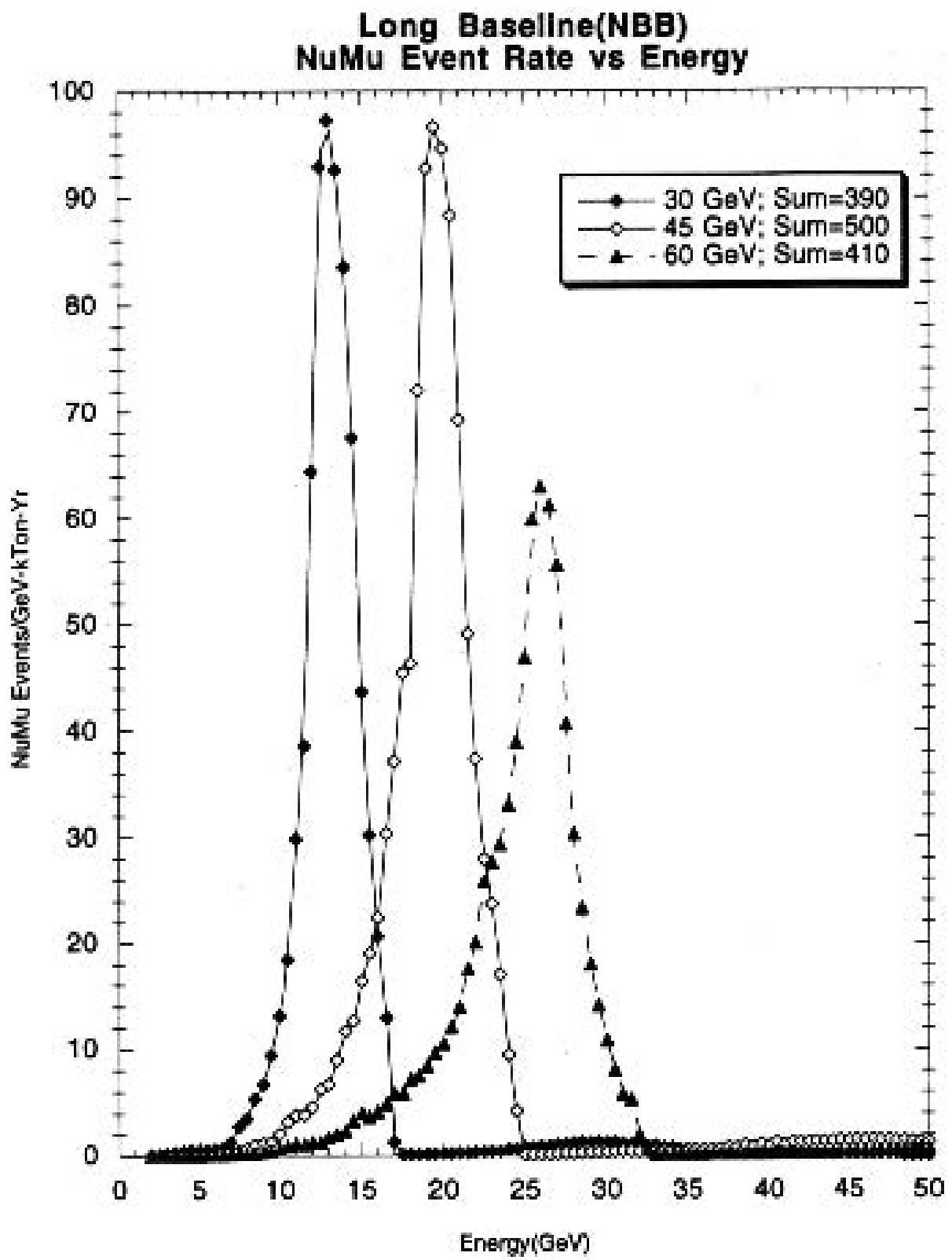

Figure 8 
Average NuTau/NuMu

Cross Section Ratio for

Maximal Mixing

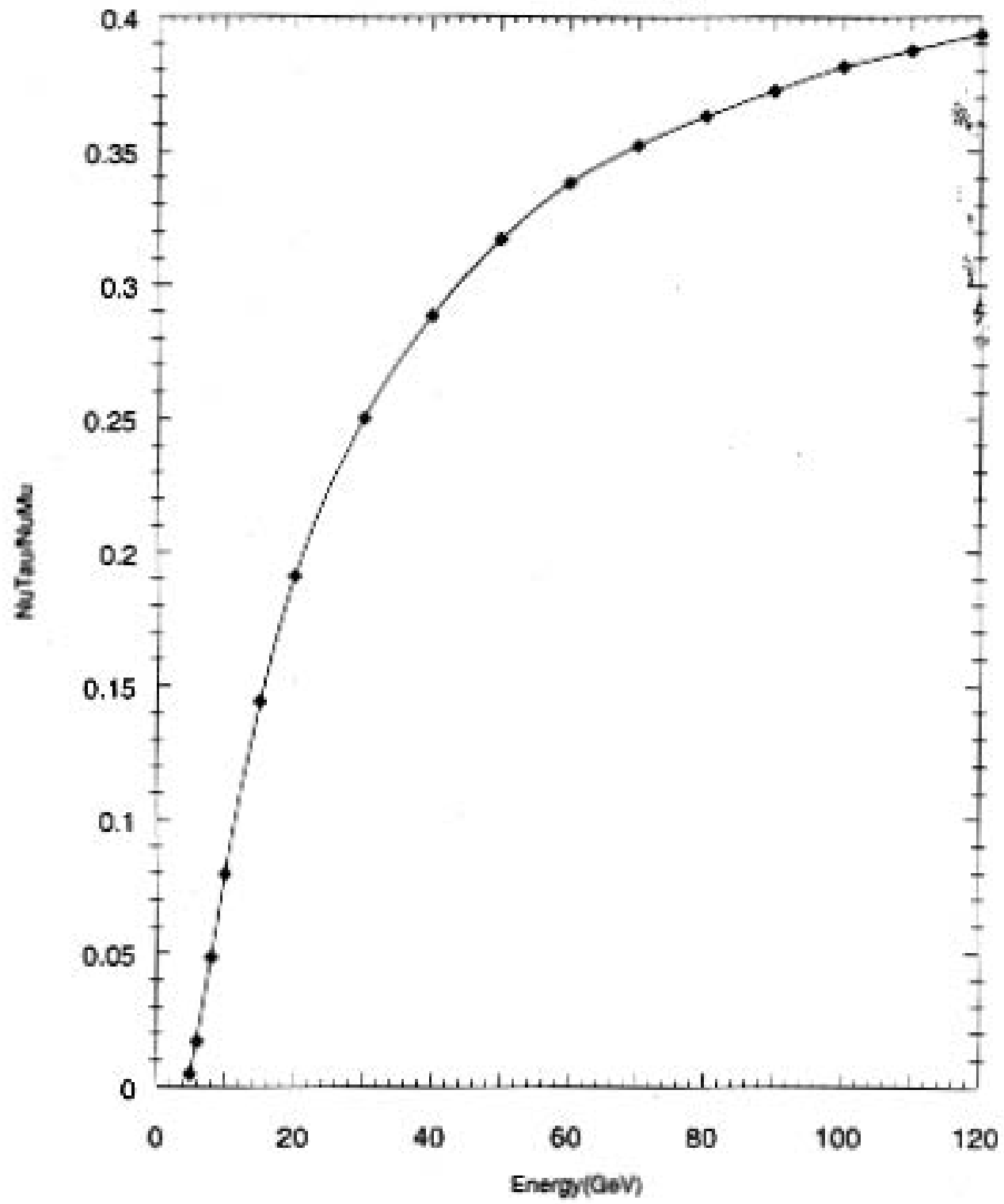

Flgure 9 
Table 1

\begin{tabular}{|c|c|c|c|c|c|}
\hline Name & $\mathrm{H} 7$ & HS & $\mathrm{H} 4$ & $\mathrm{H} 3$ & $\mathrm{H2}$ \\
\hline Horn \#1 & 4 meter & 2 meter & 2 meter & 1.5 meter & 1 meter \\
\hline Z1(meter) & 0.00 & 0.00 & 0.00 & 0.00 & 0.00 \\
\hline $\mathrm{r} 1(\mathrm{~cm})$ & 6.2 & 3.4 & 3.6 & 2.3 & 1.6 \\
\hline Z2(meter) & 2.00 & 2.00 & 2.00 & 1.50 & 1.00 \\
\hline $\mathrm{r} 2(\mathrm{~cm})$ & 1.0 & 1.0 & 1.0 & 1.0 & 1.0 \\
\hline Z3(meter) & 4.00 & 2.03 & 2.03 & 1.53 & 1.03 \\
\hline $\mathrm{r} 3(\mathrm{~cm})$ & 6.2 & 1.0 & 1.0 & 1.0 & 1.0 \\
\hline Horn $\# 2$ & 3 meter & 3 meter & 2 meter & 1.5 meter & 1 meter \\
\hline Z4(meter) & 19.00 & 20.00 & 22.00 & 22.00 & 22.00 \\
\hline $\mathrm{r} 4(\mathrm{~cm})$ & 15.6 & 17.8 & 14.5 & 11.0 & 8.2 \\
\hline 75(meter) & 22.00 & 23.00 & 24.00 & 23.50 & 23.00 \\
\hline $\mathrm{r} 5(\mathrm{~cm})$ & 2.6 & 2.5 & 2.5 & 2.5 & 2.5 \\
\hline Z6(meter) & - & 23.03 & 24.03 & 23.53 & 23.03 \\
\hline $\mathrm{r} 6(\mathrm{~cm})$ & - & 2.5 & 2.5 & 2.5 & 2.5 \\
\hline
\end{tabular}


Muon Neutrino Cross Sectlon vs. Energy $(x$ 1E-38 square $\mathrm{cm}$ )

\begin{tabular}{|c|c|c|c|c|c|}
\hline Energy & Cross Sec & Energy & Cross Sec & Energy & Cross Sec \\
\hline 1.0 & 1.0120 & 41.0 & 28.323 & 81.0 & 54.603 \\
\hline 2.0 & 1.8426 & 42.0 & 28.987 & 82.0 & 55.259 \\
\hline 3.0 & 2.5901 & 43.0 & 29.651 & 83.0 & 55.914 \\
\hline 4.0 & 3.2784 & 44.0 & 30.316 & 84.0 & 56.570 \\
\hline 5.0 & 3.9696 & 45.0 & 30.975 & 85.0 & 57.226 \\
\hline 6.0 & 4.6656 & 46.0 & 31.633 & 86.0 & 57.881 \\
\hline 7.0 & 5.3518 & 47.0 & 32.291 & 87.0 & 58.537 \\
\hline B. 0 & 6.0330 & 48.0 & 32.949 & $8 B .0$ & 59.192 \\
\hline 9.0 & 6.7230 & 49.0 & 33.607 & 89.0 & 59.848 \\
\hline 10.0 & 7.4512 & 50.0 & 34.265 & 90.0 & 60.504 \\
\hline 11.0 & B. 1793 & 51.0 & 34.923 & 91.0 & 61.159 \\
\hline 12.0 & 8.8769 & 52.0 & 35.581 & 92.0 & 61.815 \\
\hline 13.0 & 9.5712 & 53.0 & 36.239 & 93.0 & 62.471 \\
\hline 14.0 & 10.265 & 54.0 & 36.897 & 94.0 & 63.126 \\
\hline 15.0 & 10.944 & 55.0 & 37.555 & 95.0 & 63.782 \\
\hline 16.0 & 11.623 & 56.0 & 38.212 & 96.0 & 64.437 \\
\hline 17.0 & 12.303 & 57.0 & 38.868 & 97.0 & 65.093 \\
\hline 18.0 & 12.977 & 58.0 & 39.524 & 98.0 & 65.749 \\
\hline 19.0 & 13.646 & 59.0 & 40.179 & 99.0 & 66.404 \\
\hline 20.0 & 14,314 & 60.0 & 40.835 & 100.0 & 67,060 \\
\hline 21.0 & 14,982 & 61,0 & 41.490 & 101.0 & 67,716 \\
\hline 22.0 & 15.650 & 62.0 & 42.146 & 102.0 & 68.371 \\
\hline 23.0 & 16.319 & 63.0 & 42.802 & 103.0 & 69.027 \\
\hline 24.0 & 16.988 & 64.0 & 43.457 & 104.0 & 69.682 \\
\hline 25.0 & 17.656 & 65.0 & 44.113 & 105.0 & 70.338 \\
\hline 26.0 & 18.325 & 66.0 & 44.769 & 106.0 & 70.994 \\
\hline 27.0 & 18.993 & 67.0 & 45,424 & 107.0 & 71.649 \\
\hline 28.0 & 19.662 & 68.0 & 46.080 & 108.0 & 72.305 \\
\hline 29.0 & 20.330 & 69.0 & 46.736 & 109.0 & 72.961 \\
\hline 30.0 & 20.997 & 70.0 & 47.391 & 110.0 & 73.616 \\
\hline 31.0 & 21.665 & 71.0 & 48.047 & 111.0 & 74.272 \\
\hline 32.0 & 22.333 & 72.0 & 48.702 & 112.0 & 74.928 \\
\hline 33.0 & 23.001 & 73.0 & 49.358 & 113.0 & 75.583 \\
\hline 34.0 & 23.669 & 74.0 & 50.014 & 114.0 & 76.239 \\
\hline 35.0 & 24.337 & 75.0 & 50.669 & 115.0 & 76.894 \\
\hline 36.0 & 25.001 & 76.0 & 51.325 & 116.0 & 77.550 \\
\hline 37.0 & 25.665 & 77.0 & 51.981 & 117.0 & 78.206 \\
\hline 38.0 & 26.330 & 78.0 & 52.636 & 118.0 & 78.861 \\
\hline 39.0 & 26.994 & 79.0 & 53.292 & 119.0 & 79.517 \\
\hline 40.0 & 27.658 & 80.0 & 53.947 & 120.0 & 80.173 \\
\hline
\end{tabular}

